\title{
Homotopy Method for a General Multiobjective Programming Problem under Generalized Quasinormal Cone Condition
}

\author{
X. Zhao, ${ }^{1,2}$ S. G. Zhang, ${ }^{2}$ Y. T. Yang, ${ }^{1}$ and Q. H. Liu ${ }^{3}$ \\ ${ }^{1}$ Department of Mathematics, Beihua University, Jilin 132013, China \\ ${ }^{2}$ Department of Mathematics, Jilin University, Changchun 130001, China \\ ${ }^{3}$ Institute of Applied Mathematics, Changchun University of Technology, Changchun 130012, China \\ Correspondence should be addressed to Q. H. Liu, liuqinghuaij1@163.com
}

Received 14 February 2012; Accepted 24 July 2012

Academic Editor: Irena Rachůnková

Copyright (C) 2012 X. Zhao et al. This is an open access article distributed under the Creative Commons Attribution License, which permits unrestricted use, distribution, and reproduction in any medium, provided the original work is properly cited.

A combined interior point homotopy continuation method is proposed for solving general multiobjective programming problem. We prove the existence and convergence of a smooth homotopy path from almost any interior initial interior point to a solution of the KKT system under some basic assumptions.

\section{Introduction}

In this paper, for any two vectors $y=\left(y_{1}, y_{2}, \ldots, y_{n}\right)^{T}$ and $z=\left(z_{1}, z_{2}, \ldots, z_{n}\right)^{T}$ in $R^{n}$, we use the following conventions:

$$
\begin{gathered}
y=z, \quad \text { iff } y_{i}=z_{i}, i=1,2, \ldots, n ; \\
y<z, \quad \text { iff } y_{i}<z_{i}, i=1,2, \ldots, n \\
y \leqq z, \quad \text { iff } y_{i} \leq z_{i}, i=1,2, \ldots, n ; \\
y \leq z, \quad \text { iff } y_{i} \leq z_{i}, y \neq z, i=1,2, \ldots, n
\end{gathered}
$$


We consider the following multiobjective programming problem:

(MOP)

$$
\begin{array}{cl}
\min & f(x), \\
\text { s.t. } & g(x) \leqq 0, \\
& h(x)=0,
\end{array}
$$

where $f=\left(f_{1}, f_{2}, \ldots, f_{p}\right)^{T}: R^{n} \rightarrow R^{p}, g=\left(g_{1}, g_{2}, \ldots, g_{m}\right)^{T}: R^{n} \rightarrow R^{m}$, and $h=$ $\left(h_{1}, h_{2}, \ldots, h_{s}\right)^{T}: R^{n} \rightarrow R^{s}$.

For $\lambda=\left(\lambda_{1}, \lambda_{2}, \ldots, \lambda_{p}\right)^{T} \in R^{p}$, let $R_{+}^{n}$ and $R_{++}^{n}$ denote the nonnegative and positive orthant of $R^{n}$. Respectively, let

$$
\begin{gathered}
\Omega=\left\{x \in R^{n} \mid g(x) \leqq 0, h(x)=0\right\}, \quad \Omega_{0}=\left\{x \in R^{n} \mid g(x)<0, h(x)=0\right\}, \quad \partial \Omega=\frac{\Omega}{\Omega_{0}}, \\
\Lambda^{+}=\left\{\lambda \in R_{+}^{p} \mid \sum_{i=1}^{p} \lambda_{i}=1\right\}, \quad \Lambda^{++}=\left\{\lambda \in R_{++}^{p} \mid \sum_{i=1}^{p} \lambda_{i}=1\right\}, \\
I=\{1,2, \ldots, m\}, \quad J=\{1,2, \ldots, s\},
\end{gathered}
$$

and let

$$
B(x)=\left\{i \in\{1,2, \ldots, m\} \mid g_{i}(x)=0\right\}
$$

denote the active index set at a given point.

MOP has important application in many practical fields like production planning, structural designing, portfolio selection, and so forth. Research on it can be traced back to Pareto [1], Von Neumann and Morgenstern [2], and Koopmans [3] or even earlier. Especially, more and more attention has been paid to the homotopy method since Kellogg et al. [4], Smale [5], and Chow et al. [6] published the remarkable papers. The homotopy method now becomes an important tool for numerically solving complementary, variational inequalities, convex multiobjective programming, and nonlinear mathematical programming et al. [7-12] as a globally convergent method.

Among many methods, the weighed sum method is popular and efficient. It transforms the MOP to a single-objective programming [13]:

$$
\begin{array}{cc}
\min & \lambda^{T} f(x), \\
\text { s.t. } & g(x) \leqq 0, \\
& \sum_{i=1}^{p} \lambda_{i}=1,
\end{array}
$$

where $\lambda$ is the weight vector. 
Recently, Song and Yao [14] generalize the combined homotopy interior point method to the general multi-objective programming problem under the so-called normal cone condition instead of the convexity condition about the feasible set. In that paper, they proved the existence of the homotopy path under the following assumptions:

$\left(\mathrm{A}_{1}\right) \Omega_{0}$ is nonempty and bounded;

$\left(\mathrm{A}_{2}\right)$ for all $x \in \Omega$, the vectors $\left\{\nabla g_{i}(x), i \in B(x), \nabla h_{j}(x), j \in J\right\}$ are linearly independent;

$\left(\mathrm{A}_{3}\right)$ for all $x \in \Omega,\left\{x+\sum_{i \in B(x)} u_{i} \nabla g_{i}(x)+\sum_{j \in J} z_{j} \nabla h_{j}(x): z_{j} \in R, u_{i} \geq 0, j \in J, i \in\right.$ $B(x)\} \cap \Omega=\{x\}$.

In [14], the combined homotopy method was given as follows:

$$
\bar{H}\left(\omega, \omega^{(0)}, t\right)=\left[\begin{array}{c}
(1-t)(\nabla f(x) \lambda+\nabla g(x) u)+\nabla h(x) z+t\left(x-x^{(0)}\right) \\
h(x) \\
U \times g(x)-t U^{(0)} \times g\left(x^{(0)}\right) \\
(1-t)\left(1-\sum_{i=1}^{p} \lambda_{i}\right) e-t\left(\lambda-\lambda^{(0)}\right)
\end{array}\right]=0
$$

where $x^{(0)} \in \Omega_{0}, u^{(0)}>0, \lambda^{(0)}>0$, and $\sum_{i=1}^{p} \lambda_{i}{ }^{(0)}=1$. However, the solution simply yields $\lambda=\lambda^{(0)}$ for all $t \in(0,1]$. That is, $\lambda$ is fixed. In fact, from the last equation, we have $p(1-t)+$ $(p t-p-t) \sum_{i=1}^{p} \lambda_{i}+t=0$. According to this, we know that $\lambda \equiv \lambda^{0}$ for all of $t \in[0,1]$.

That is, these methods are all solving the single-objective programming problem.

In [15], they present the concept of "positive linear independent" and weaken the assumptions than the ones in [14]. But in order to extend their results to a broader class of nonconvex multi-objective programming problems, we construct a new homotopy equation under generalized quasinorm cone condition in this paper and $\lambda$ is not fixed in the calculation process.

The paper is organized as follows. In Section 2, we recall some notations and preliminaries results. In Section 3, we construct a new combined homotopy mapping and prove the existence and convergence of a smooth homotopy path from almost any interior initial point to the KKT points of MOP under some assumptions. In Section 4, numerical results are given, which show that the method is feasible and effective.

\section{Some Definitions and Properties}

Definition 2.1. Let $U \subset R^{n}$ be an open set, and let $\varphi: U \rightarrow R^{P}$ be a smooth mapping. If Range $[\partial \varphi(x) / \partial x]=R^{p}$ for all $x \in \varphi^{-1}(y)$, then $y \in R^{p}$ is a regular value and $x \in R^{n}$ is a regular point.

Definition 2.2. Let $\eta_{i}: R^{n} \rightarrow R^{n}(i=1,2, \ldots, m)$ and $\beta_{j}: R^{n} \rightarrow R^{n}(j=1,2, \ldots, s)$. For any $x \in \Omega,\left\{\nabla g_{i}(x), \eta_{i}(x): i \in B(x)\right\}$ is said to be positive linear independent with respect to $\beta(x)$, if

$$
\beta(x) z+\sum_{i \in B(x)}\left(y_{i} \nabla g_{i}(x)+u_{i} \eta_{i}(x)\right)=0, \quad z \in R^{s}, y_{i} \geq 0, u_{i} \geq 0
$$


implies that

$$
z=0, \quad y_{i}=0, \quad u_{i}=0 \quad(i \in B(x)),
$$

where $\beta(x)=\left(\beta_{1}(x), \ldots, \beta_{s}(x)\right)$.

Lemma 2.3 (parametric form of the Sard theorem on a smooth manifold; see [16]). Let $Q, N, P$ be smooth manifolds of dimensions $q, m, p$. Respectively, let $\varphi: Q \times N \rightarrow P$ be a $C^{r}$ map, where $r>\max \{0, m-p\}$. If $0 \in P$ is a regular value of $\varphi$, then for almost all $\alpha \in Q, 0$ is a regular value of $\varphi(\alpha, \cdot)$.

Lemma 2.4 (inverse image theorem; see [17]). If 0 is a regular value of the mapping $\varphi_{\alpha}(\cdot) \triangleq$ $\varphi(\alpha, \cdot)$, then $\varphi_{\alpha}^{-1}(0)$ consists of some smooth manifolds.

Lemma 2.5 (classification theorem of one-dimensional manifold; see [17]). A one-dimensional smooth manifold is diffeomorphic to a unit circle or a unit interval.

The following four basic assumptions are commonly used in this paper:

$\left(C_{1}\right) \Omega_{0}$ is nonempty and bounded;

$\left(C_{2}\right)$ for any $x \in \Omega$ and $t \in[0,1]$, there exists map $\eta(x)$ and $\beta(x)$, such that $\left\{\nabla g_{i}(x)\right.$, $\left.\eta_{i}(x): i \in B(x)\right\}$ is positive linear independent with respect to $\nabla h(x)+t(\beta(x)-$ $\nabla h(x))$;

$\left(C_{3}\right)$ for any $x \in \partial \Omega$,

$$
\left\{x+\sum_{i \in B(x)} u_{i} \eta_{i}(x)+\beta(x) z: z \in R^{s}, u_{i} \geq 0, i \in B(x)\right\} \bigcap \Omega=\{x\}
$$

(generalized quasinormal cone condition);

$\left(\mathrm{C}_{4}\right)$ for any $x \in \Omega, \nabla h(x)^{T} \beta(x)$ is nonsingular.

Remark 2.6. If $\Omega$ satisfies the assumptions $\left(\mathrm{A}_{1}\right)-\left(\mathrm{A}_{3}\right)$, then it necessarily satisfies the assumptions $\left(\mathrm{C}_{1}\right)-\left(\mathrm{C}_{4}\right)$.

In fact, if we choose $\eta(x)=\nabla g(x)$ and $\beta(x)=\nabla h(x)$, then it is easy to get the result. Clearly, if $\Omega$ satisfies the assumptions $\left(C_{1}\right)-\left(C_{4}\right)$, then it does not necessarily satisfies the assumptions $\left(\mathrm{A}_{1}\right)-\left(\mathrm{A}_{3}\right)$.

\section{Main Results}

Let $x \in \Omega$ be a KKT point of MOP; our aim is to find $(\lambda, u, z) \in R_{+}^{p+m} \times R^{s}$, such that

$$
\begin{gathered}
\nabla f(x) \lambda+\nabla g(x) u+\nabla h(x) z=0, \\
U g(x)=0, \\
1-\sum_{i=1}^{p} \lambda_{i}=0,
\end{gathered}
$$


where $\nabla f(x)=\left(\nabla f_{1}(x), \ldots, \nabla f_{p}(x)\right) \in R^{n \times p}, \nabla g(x)=\left(\nabla g_{1}(x), \ldots, \nabla g_{m}(x)\right) \in R^{n \times m}, \nabla h(x)=$ $\left(\nabla h_{1}(x), \ldots, \nabla h_{m}(x)\right) \in R^{n \times s}$.

Meanwhile, the KKT system of MOP is (3.1a)-(3.1c).

For a convex multi-objective programming problem, the solution of the MOP can be obtained from the KKT system. And for a nonconvex multi-objective programming problem, it is significant that we can obtain a solution of the KKT system.

To solve the KKT system (3.1a)-(3.1c), we construct a homotopy equation as follows:

$$
\begin{aligned}
& H\left(\omega, \omega^{(0)}, t\right) \\
& =\left[\begin{array}{c}
(1-t)\left(\nabla f(x) \lambda+\nabla g(x) u+t \eta(x) u^{2}\right)+[\nabla h(x)+t(\beta(x)-\nabla h(x))] z+t\left(x-x^{(0)}\right) \\
h(x) \\
U \times g(x)-t U^{(0)} \times g\left(x^{(0)}\right) \\
(1-t)\left(1-\sum_{i=1}^{p} \lambda_{i}\right) e-t\left(\lambda^{5 / 2}-\left(\lambda^{(0)}\right)^{5 / 2}\right)
\end{array}\right] \\
& =0
\end{aligned}
$$

where $\omega^{(0)}=\left(x^{(0)}, \lambda^{(0)}, u^{(0)}, z^{(0)}\right) \in \Omega_{0} \times \Lambda^{++} \times R_{++}^{m} \times\{0\}, \omega=(x, \lambda, u, z) \in \Omega \times R^{p+m+s}, u^{2}=$ $\left(u_{1}^{2}, u_{2}^{2} \ldots, u_{m}^{2}\right)^{T} \in R^{m}, \lambda^{5 / 2}=\left(\lambda_{1}^{5 / 2}, \lambda_{2}^{5 / 2}, \ldots, \lambda_{p}^{5 / 2}\right)^{T} \in R^{p}, U=\operatorname{diag}(u), e=(1,1, \ldots, 1)^{T} \in R^{p}$, and $t \in[0,1]$.

As $t=1$, the homotopy equation (3.2) becomes

$$
\begin{gathered}
\beta(x) z+\left(x-x^{(0)}\right)=0, \\
h(x)=0 \\
U g(x)-U^{(0)} g\left(x^{(0)}\right)=0, \\
\lambda^{5 / 2}=\left(\lambda^{(0)}\right)^{5 / 2} .
\end{gathered}
$$

By the assumption $\left(C_{3}\right)$, we get $z=0, x=x^{(0)}$. Since $g\left(x^{(0)}\right)<0$ and $x=x^{(0)},(3.3 \mathrm{c})$ implies that $u=u^{(0)}$. Equation (3.3d) shows that $\lambda=\lambda^{(0)}$. That is, $H\left(\omega, \omega^{(0)}, 1\right)=0$ with respect to $\omega$ has only one solution $\omega=\omega^{(0)}=\left(x^{(0)}, \lambda^{(0)}, u^{(0)}, 0\right)$.

As $t=0, H\left(\omega, \omega^{(0)}, t\right)=0$ turns to the KKT system (3.1a)-(3.1c).

For a given $\omega^{(0)}$, rewrite $H\left(\omega, \omega^{(0)}, t\right)$ as $H_{\omega^{(0)}}(\omega, t)$. The zero-point set of $H_{\omega^{(0)}}$ is

$$
H_{\omega^{(0)}}^{-1}=\left\{(\omega, t) \in \Omega \times R_{++}^{p+m} \times R^{S} \times(0,1]: H\left(\omega, \omega^{(0)}, t\right)=0\right\} .
$$

Theorem 3.1. Suppose $f, g$, and $h$ are three times continuous differentiable functions. In addition, let the assumptions $\left(C_{1}\right)-\left(C_{2}\right)$ hold and $\eta_{i}, \beta_{j}$ twice times continuously differentiable functions. Then for 
almost all initial points $\omega^{(0)} \in \Omega_{0} \times \Lambda^{++} \times R_{++}^{m} \times\{0\}, 0$ is a regular value of $H_{\omega^{(0)}}$ and $H_{\omega^{(0)}}^{-1}$ consists of some smooth curves. Among them, a smooth curve, say $\Gamma_{\omega^{(0)}}$, is starting from $\left(\omega^{(0)}, 1\right)$.

Proof. Denote the Jacobi matrix of $H\left(\omega, \omega^{(0)}, t\right)$ by $D H\left(\omega, \omega^{(0)}, t\right)$. For any $\omega^{(0)} \in \Omega_{0} \times \Lambda^{++} \times$ $R_{++}^{m} \times\{0\}$ and $t \in[0,1]$, we have $D H\left(\omega, \omega^{(0)}, t\right)=\left(\partial H / \partial \omega, \partial H / \partial \omega^{(0)}, \partial H / \partial t\right)$. Now, we consider the submatrix of $D H\left(\omega, \omega^{(0)}, t\right)$.

For any $\left(x, x^{(0)}, \lambda^{(0)}, u^{(0)}\right) \in R^{n} \times \Omega_{0} \times \Lambda^{++} \times R_{++}^{m}$,

$$
\frac{\partial H}{\partial\left(x, x^{(0)}, \lambda^{(0)}, u^{(0)}\right)}=\left[\begin{array}{cccc}
Q & -t I_{n} & 0 & 0 \\
\nabla h(x)^{T} & 0 & 0 & 0 \\
U \nabla g(x)^{T} & -t U^{(0)} \nabla g\left(x^{(0)}\right)^{T} & 0 & -t \operatorname{diag}\left(g\left(x^{(0)}\right)\right) \\
0 & 0 & \frac{5}{2} t\left(\lambda^{(0)}\right)^{3 / 2} I_{p} & 0
\end{array}\right]
$$

where $Q=(1-t)\left(\sum_{i=1}^{p} \lambda_{i} \nabla^{2} f_{i}(x)+\sum_{j=1}^{m} u_{j} \nabla^{2} g_{j}(x)+t \sum_{j=1}^{m} u_{j}^{2} \nabla \eta_{j}(x)\right)+\left[\nabla^{2} h(x)+t(\nabla \beta(x)-\right.$ $\left.\left.\nabla^{2} h(x)\right)\right] z+t I_{n}$.

We obtain that

$$
\operatorname{rank} \frac{\partial H}{\partial\left(x, x^{(0)}, \lambda^{(0)}, u^{(0)}\right)}=n+p+m+s
$$

That is, 0 is a regular value of $H$. By parametric form of the Sard theorem, for almost all $\omega^{(0)} \in \Omega_{0} \times \Lambda^{++} \times R_{++}^{m} \times\{0\}, 0$ is a regular value of $H_{\omega^{(0)}}$. By inverse image theorem, $H_{\omega^{(0)}}^{-1}(0)$ consists of some smooth curves. Since $H\left(\omega^{(0)}, \omega^{(0)}, 1\right)=0$, there must be a smooth curve, denoted by $\Gamma_{\omega^{(0)}}$, that starts from $\left(\omega^{(0)}, 1\right)$.

Theorem 3.2. Let assumptions $\left(C_{1}\right)-\left(C_{2}\right)$ hold. For a given $\omega^{(0)}=\left(x^{(0)}, \lambda^{(0)}, u^{(0)}, z^{(0)}\right) \in \Omega_{0} \times \Lambda^{++} \times$ $R_{++}^{m} \times\{0\}$, if 0 is a regular value of $H_{\omega^{(0)}}$, then the projection of the smooth curve $\Gamma_{\omega^{(0)}}$ on the component $\lambda$ is bounded.

Proof. Suppose that the conclusion does not hold. Since $(0,1]$ is bounded, there exists a sequence $\left\{\left(\omega^{(k)}, t_{k}\right)\right\} \subset \Gamma_{\omega^{(0)}}$, such that

$$
t_{k} \longrightarrow t_{* \prime} \quad\left\|\lambda^{(k)}\right\| \longrightarrow \infty
$$

From the last equality of (3.2), we have

$$
\left(\begin{array}{c}
1-t_{k} \\
1-t_{k} \\
\cdots \\
1-t_{k}
\end{array}\right)-\left(\begin{array}{c}
\left(1-t_{k}\right) \lambda_{1}^{(k)}+\left(1-t_{k}\right) \sum_{i \neq 1} \lambda_{i}^{(k)}+t_{k}\left(\lambda_{1}^{(k)}\right)^{5 / 2} \\
\left(1-t_{k}\right) \lambda_{2}^{(k)}+\left(1-t_{k}\right) \sum_{i \neq 2} \lambda_{i}^{(k)}+t_{k}\left(\lambda_{2}^{(k)}\right)^{5 / 2} \\
\ldots \\
\left(1-t_{k}\right) \lambda_{p}^{(k)}+\left(1-t_{k}\right) \sum_{i \neq p} \lambda_{i}^{(k)}+t_{k}\left(\lambda_{p}^{(k)}\right)^{5 / 2}
\end{array}\right)-t_{k}\left(\begin{array}{c}
\left(\lambda_{1}^{(0)}\right)^{5 / 2} \\
\left(\lambda_{2}^{(0)}\right)^{5 / 2} \\
\ldots \\
\left(\lambda_{p}^{(0)}\right)^{5 / 2}
\end{array}\right)=0
$$


If we assume $\left\|\lambda^{(k)}\right\| \rightarrow+\infty(k \rightarrow \infty)$, this hypothesis implies

$$
\left\{i \in\{1,2, \ldots, p\}: \lim _{k \rightarrow \infty} \lambda_{i}^{(k)}=\infty\right\} \neq \Phi
$$

Since $t_{k} \rightarrow t_{*}, \lambda^{(k)}>0$, it follows that the second part in the left-hand side of some equations in (3.8) tends to infinity as $k \rightarrow \infty$. But the other two parts are bounded. This is impossible. Thus, the component $\lambda$ is bounded.

Theorem 3.3. Let $f, g$, and $h$ be three times continuous differentiable functions. In addition, let the assumptions $\left(C_{1}\right)-\left(C_{4}\right)$ hold and $\eta_{i}, \beta_{j}$ twice times continuously differentiable functions. Then, for almost all of $\omega^{(0)} \in \Omega_{0} \times \Lambda^{++} \times R_{++}^{m} \times\{0\}, H_{\omega^{(0)}}^{-1}(0)$ contains a smooth curve $\Gamma_{\omega^{(0)}} \subset \Omega \times R_{+}^{p} \times R_{+}^{m} \times$ $R^{s} \times(0,1]$, which starts from $\left(\omega^{(0)}, 1\right)$. As $t \rightarrow 0$, the limit set $T \times\{0\} \subset \Omega \times \Lambda^{+} \times R_{+}^{m} \times R^{s} \times\{0\}$ of $\Gamma_{\omega^{(0)}}$ is nonempty and every point in $T$ is a solution of the KKT system (3.1a)-(3.1c).

Proof. From the homotopy equation (3.2), it is easy to see that $\Gamma_{\omega^{(0)}} \subset \Omega \times R_{+}^{p} \times R_{+}^{m} \times R^{s} \times(0,1]$. By Theorem 3.1, for almost all $\omega^{(0)} \in \Omega_{0} \times \Lambda^{++} \times R_{++}^{m} \times\{0\}, 0$ is a regular value of $H_{\omega^{(0)}}$ and $H_{\omega^{(0)}}^{-1}$ contains a smooth curve $\Gamma_{\omega^{(0)}}$ starting from $\left(\omega^{(0)}, 1\right)$. By the classification theorem of one-dimensional smooth manifolds, $\Gamma_{\omega^{(0)}}$ is diffeomorphic to a unit circle or the unit interval $(0,1]$.

Noticing that

$$
\begin{aligned}
\left|\frac{\partial H_{\omega^{(0)}}\left(\omega^{(0)}, 1\right)}{\partial \omega}\right| & =\left|\begin{array}{cccc}
I_{n} & 0 & 0 & \beta\left(x^{(0)}\right) \\
\nabla h\left(x^{(0)}\right)^{T} & 0 & 0 & 0 \\
U^{(0)} \nabla g\left(x^{(0)}\right)^{T} & 0 & \operatorname{diag}\left(g\left(x^{(0)}\right)\right) & 0 \\
0 & -\frac{5}{2}\left(\lambda^{(0)}\right)^{3 / 2} I_{p} & 0 & 0
\end{array}\right| \\
& =(-1)^{s}\left|\operatorname{diag}\left(g\left(x^{(0)}\right)\right)\right|\left|-\frac{5}{2}\left(\lambda^{(0)}\right)^{3 / 2} I_{p}\right|\left|\nabla h\left(x^{(0)}\right)^{T} \beta\left(x^{(0)}\right)\right| .
\end{aligned}
$$

Because $g\left(x^{(0)}\right)<0, \lambda^{(0)} \in \Lambda^{++}$and by the assumption $\left(\mathrm{C}_{4}\right)$, we know that $\left[\partial H_{\omega^{(0)}}\left(\omega^{(0)}, 1\right) / \partial \omega\right]$ is nonsingular. Therefore, the smooth curve $\Gamma_{\omega^{(0)}}$ starts from $\left(\omega^{(0)}, 1\right)$ diffeomorphic to $(0,1]$.

Let $\left(\omega^{*}, t_{*}\right)$ be a limit point of $\Gamma_{\omega^{(0)}}$; only three cases are possible:

(a) $\left(\omega^{*}, t_{*}\right) \in \Omega \times \Lambda^{+} \times R_{+}^{m} \times R^{s} \times\{0\}$;

(b) $\left(\omega^{*}, t_{*}\right) \in \partial\left(\Omega_{0} \times R_{+}^{p+m}\right) \times R^{s} \times(0,1]$;

(c) $\left(\omega^{*}, t_{*}\right) \in \Omega \times R_{+}^{p+m} \times R^{s} \times\{1\}$.

Because $H\left(\omega^{(0)}, \omega^{(0)}, 1\right)=0$ has a unique solution $\left(\omega^{(0)}, 1\right)$, the case (c) will not happen. In case $(b)$, because $\Omega$ and $(0,1]$ are bounded sets and by the assumption $\left(C_{2}\right)$, for any $x \in \Omega$ and $t \in[0,1]$, there exists map $\eta(x)$ and $\beta(x)$ such that, $\left\{\nabla g_{i}(x), \eta_{i}(x): i \in B(x)\right\}$ is positive linear independent with respect to $\nabla h(x)+t(\beta(x)-\nabla h(x))$. From the first equation of (3.2), we get that the component $z$ of $\Gamma_{\omega^{(0)}}$ is bounded. 
If case (b) holds, then there exists a sequence $\left\{\left(\omega^{(k)}, t_{k}\right)\right\} \subset \Gamma_{\omega^{(0)}}$, such that

$$
\left\|\left(\omega^{(k)}, t_{k}\right)\right\| \rightarrow \infty
$$

Because $\Omega$ and $(0,1]$ are bounded, there exists a subsequence (denoted also by $\left.\left\{\left(\omega^{(k)}, t_{k}\right)\right\} \subset \Gamma_{\omega^{(0)}}\right)$ such that

$$
x^{(k)} \longrightarrow x^{*}, \quad \lambda^{(k)} \longrightarrow \lambda^{*}, \quad\left\|u^{(k)}\right\| \longrightarrow \infty, \quad z^{(k)} \longrightarrow z^{*}, \quad t_{k} \longrightarrow t_{* \prime} \quad \text { as } k \longrightarrow \infty
$$

By the third equation of (3.2), we have

$$
g\left(x^{(k)}\right)=t_{k}\left(U^{(k)}\right)^{-1} U^{(0)} g\left(x^{(0)}\right)
$$

Hence, the active index set $B\left(x^{*}\right)$ is nonempty.

From the first equation of (3.2), it follows that

$$
\begin{aligned}
& \left(1-t_{k}\right)\left(\nabla f\left(x^{(k)}\right) \lambda^{(k)}+\nabla g\left(x^{(k)}\right) u^{(k)}+t_{k} \eta\left(x^{(k)}\right)\left(u^{(k)}\right)^{2}\right) \\
& +\left[\nabla h\left(x^{(k)}\right)+t_{k}\left(\beta\left(x^{(k)}\right)-\nabla h\left(x^{(k)}\right)\right)\right] z^{(k)}+t_{k}\left(x^{(k)}-x^{(0)}\right)=0 .
\end{aligned}
$$

(i) When $t_{*}=1$, rewrite (3.14) as

$$
\begin{gathered}
\sum_{j \in B\left(x^{*}\right)}\left(1-t_{k}\right)\left(\nabla g_{j}\left(x^{(k)}\right) u_{j}^{(k)}+t_{k} \eta_{j}\left(x^{(k)}\right)\left(u_{j}^{(k)}\right)^{2}\right) \\
+t_{k} \beta\left(x^{(k)}\right) z^{(k)}+\left(1-t_{k}\right) \nabla h\left(x^{(k)}\right) z^{(k)}+\left(x^{(k)}-x^{(0)}\right) \\
=-\left(1-t_{k}\right)\left[\sum_{j \notin B\left(x^{*}\right)} \nabla g_{j}\left(x^{(k)}\right) u_{j}^{(k)}+t_{k} \eta_{j}\left(x^{(k)}\right)\left(u_{j}^{(k)}\right)^{2}\right. \\
\left.+\nabla f\left(x^{(k)}\right) \lambda^{(k)}-\left(x^{(k)}-x^{(0)}\right)\right] .
\end{gathered}
$$

From the fact that $u_{j}^{(k)}$ is bounded for $j \notin B\left(x^{*}\right)$ and by the assumptions $\left(C_{1}\right)$ and $\left(C_{2}\right)$, when $k \rightarrow \infty$, we observe that

$$
\sum_{j \in B\left(x^{*}\right)}\left(\nabla g_{j}\left(x^{*}\right) \lim _{k \rightarrow \infty}\left(1-t_{k}\right) u_{j}^{(k)}+\eta_{j}\left(x^{*}\right) \lim _{k \rightarrow \infty}\left(1-t_{k}\right) t_{k}\left(u_{j}^{(k)}\right)^{2}\right)=-\left(\beta\left(x^{*}\right) z^{*}+x^{*}-x^{(0)}\right)
$$


It is easy to see that the right-hand side of the equation is bounded. By the assumption $\left(C_{2}\right)$, we have

$$
\lim _{k \rightarrow \infty}\left(1-t_{k}\right) u_{j}^{(k)}=0, \quad \lim _{k \rightarrow \infty}\left(1-t_{k}\right) t_{k}\left(u_{j}^{(k)}\right)^{2}=\alpha_{j}, \quad j \in B\left(x^{*}\right),
$$

where $\alpha_{j} \geq 0$.

Then, we have

$$
x^{(0)}=x^{*}+\beta\left(x^{*}\right) z^{*}+\sum_{j \in B\left(x^{*}\right)} \alpha_{j} \eta_{j}\left(x^{*}\right)
$$

which contradicts the assumption $\left(\mathrm{C}_{3}\right)$.

(ii) When $t_{*} \in[0,1)$, rewrite $(3.14)$ as

$$
\begin{aligned}
\sum_{j \in B\left(x^{*}\right)} & \left(1-t_{k}\right)\left(\nabla g_{j}\left(x^{(k)}\right) u_{j}^{(k)}+t_{k} \eta_{j}\left(x^{(k)}\right)\left(u_{j}^{(k)}\right)^{2}\right) \\
= & -\left(1-t_{k}\right)\left[\sum_{j \notin B\left(x^{*}\right)}\left(\nabla g_{j}\left(x^{(k)}\right) u_{j}^{(k)}+t_{k} \eta_{j}\left(x^{(k)}\right)\left(u_{j}^{(k)}\right)^{2}\right)+\nabla f\left(x^{(k)}\right) \lambda^{(k)}\right] \\
& -t_{k}\left(x^{(k)}-x^{(0)}\right)-\left[\nabla h\left(x^{(k)}\right)+t_{k}\left(\beta\left(x^{(k)}\right)-\nabla h\left(x^{(k)}\right)\right)\right] z^{(k)}
\end{aligned}
$$

We know that, since $\Omega$ and $u_{j}^{(k)}, j \notin B\left(x^{*}\right)$ are bounded as $k \rightarrow \infty$, the right-hand side of (3.19) is bounded. But by the assumption $\left(C_{2}\right)$, if $u_{j}^{(k)} \rightarrow \infty\left(j \in B\left(x^{*}\right)\right)$, then the left-hand side of (3.19) is infinite; this is a contradiction.

As a conclusion, (a) is the only possible case, and $\omega^{*}$ is a solution of the KKT system.

Let $s$ be the arc-length of $\Gamma_{\omega^{(0)}}$. We can parameterize $\Gamma_{\omega^{(0)}}$ with respect to $s$.

Theorem 3.4. The homotopy path $\Gamma_{\omega^{(0)}}$ is determined by the following initial-value problem for the ordinary differential equation

$$
\begin{gathered}
D H_{\omega^{(0)}}(\omega(s), t(s))\left[\begin{array}{c}
\cdot \\
\omega \\
\cdot \\
\mu
\end{array}\right]=0, \\
\omega(0)=\omega^{(0)}, \quad t(0)=1 .
\end{gathered}
$$

The component $\omega^{*}$ of the solution point $\left(\omega\left(s^{*}\right), t\left(s^{*}\right)\right)$, for $t\left(s^{*}\right)=0$, is the solution of the KKT system.

\section{Algorithm and Numerical Example}

Algorithm 4.1. MOP's Euler-Newton method. 
Step 1. Give an initial point $\left(\omega^{(0)}, 1\right) \in \Omega_{0} \times R_{++}^{p+m} \times\{0\} \times\{1\}$, an initial step length $h_{0}>0$, and three small positive numbers $\varepsilon_{1}, \varepsilon_{2}, \varepsilon_{3}$. Let $k:=0$.

Step 2. Compute the direction $\gamma^{(k)}$ of the predictor step.

(a) Compute a unit tangent vector $\xi^{(k)} \in R^{n+p+m+s+1}$ of $\Gamma_{\omega^{0}}$ at $\left(\omega^{(0)}, t_{k}\right)$.

(b) Determine the direction $\gamma^{(k)}$ of the predictor step.

If the sign of the determinant $\left|\begin{array}{c}D H_{\omega^{(0)}\left(\omega^{(k)}, t_{k}\right)}^{(k)^{T}} \\ \xi^{(k)}\end{array}\right|$ is $(-1)^{p+m+s+p m+p s+m s+1}$, take $\gamma^{(k)}=\xi^{(k)}$.

If the sign of the determinant $\left|\begin{array}{c}D H_{\omega^{(0)}\left(\omega^{(k)}, t_{k}\right)} \\ \xi^{(k)^{T}}\end{array}\right|$ is $(-1)^{p+m+s+p m+p s+m s}$, take $\gamma^{(k)}=-\xi^{(k)}$.

Step 3. Compute a corrector point $\left(\omega^{(k+1)}, t_{k+1}\right)$ :

$$
\begin{gathered}
\left(\left(\omega^{*}\right)^{(k)},\left(t_{*}\right)_{k}\right)=\left(\omega^{(k)}, t_{k}\right)+h_{k} r^{(k)}, \\
\left(\omega^{(k+1)}, t_{k+1}\right)=\left(\left(\omega^{*}\right)^{(k)},\left(t_{*}\right)_{k}\right)-D H_{\omega^{(0)}}\left(\left(\omega^{*}\right)^{(k)},\left(t_{*}\right)_{k}\right)^{+} H_{\omega^{(0)}}\left(\left(\omega^{*}\right)^{(k)},\left(t_{*}\right)_{k}\right),
\end{gathered}
$$

where

$$
D H_{\omega^{(0)}}(\omega, t)^{+}=D H_{\omega^{(0)}}(\omega, t)^{T}\left(D H_{\omega^{(0)}}(\omega, t) D H_{\omega^{(0)}}(\omega, t)^{T}\right)^{-1}
$$

is the Moore-Penrose inverse of $D H_{\omega^{(0)}}(\omega, t)$.

If $\left\|H_{\omega^{(0)}}\left(\omega^{(k+1)}, t_{k+1}\right)\right\| \leq \varepsilon_{1}$, let $h_{k+1}=\min \left\{h_{0}, 2 h_{k}\right\}$, and go to Step 4 .

If $\left\|H_{\omega^{(0)}}\left(\omega^{(k+1)}, t_{k+1}\right)\right\| \in\left(\varepsilon_{1}, \varepsilon_{2}\right)$, let $h_{k+1}=h_{k}$, and go to Step 4 .

If $\left\|H_{\omega^{(0)}}\left(\omega^{(k+1)}, t_{k+1}\right)\right\| \geq \varepsilon_{2}$, let $h_{k+1}=\max \left\{(1 / 2) h_{0},(1 / 2) h_{k}\right\}$, and go to Step 3 .

Step 4. If $\omega^{(k+1)} \in \Omega \times R_{+}^{p+m} \times R^{s}$ and $t_{k+1}>\varepsilon_{3}$, let $k=k+1$ and go to Step 2 .

If $\omega^{(k+1)} \in \Omega \times R_{+}^{p+m} \times R^{s}$ and $t_{k+1}<-\varepsilon_{3}$, let $h_{k}:=h_{k}\left(t_{k} /\left(t_{k}-t_{k+1}\right)\right)$, go to Step 3, and recompute $\left(\omega^{(k+1)}, t_{k+1}\right)$ for the initial point $\left(\omega^{(k)}, t_{k}\right)$.

If $\omega^{(k+1)} \notin \Omega \times R_{+}^{p+m} \times R^{s}$, let $h_{k}:=\left(h_{k} / 2\right)\left(t_{k} /\left(t_{k}-t_{k+1}\right)\right)$, go to Step 3, and recompute $\left(\omega^{(k+1)}, t_{k+1}\right)$ for the initial point $\left(\omega^{(k)}, t_{k}\right)$.

If $\omega^{(k+1)} \in \Omega \times R_{+}^{p+m} \times R^{s}$, and $\left|t_{k+1}\right| \leq \varepsilon_{3}$, then stop.

Example 4.2 (see [9]). Consider

$$
\begin{array}{ll}
\min & f=\min \left\{x_{1}^{2}+x_{2}^{2}+x_{3}^{2}+x_{4}^{2}+x_{5}^{2}, 3 x_{1}+2 x_{2}-\frac{1}{3} x_{3}+0.01\left(x_{4}-x_{5}\right)^{3}\right\} ; \\
\text { s.t. } & g_{1}(x)=x_{1}^{2}+x_{2}^{2}+x_{3}^{2}+x_{4}^{2}-10 \leq 0 ; \\
& h_{1}(x)=4 x_{1}-2 x_{2}+0.8 x_{3}+0.6 x_{4}+0.5 x_{5}^{2}=0 ; \\
& h_{2}(x)=x_{1}+2 x_{2}-x_{3}-0.5 x_{4}+x_{5}-2=0 .
\end{array}
$$

The results are shown in Table 1. 
Table 1: Results for Example 4.2.

\begin{tabular}{lccc}
\hline$x^{(0)}$ & $x^{*}$ & $f\left(x^{*}\right)$ & $\left\|H_{\omega^{*}}\right\|$ \\
\hline$(1.0000,2.0000,0.0000,1.0000,1.0000)$ & $(-1.3074,-2.8605,-1.0470$, & $(11.3566,-9.2942)$ & $1.0 e^{-012}$ \\
& $0.4103,0.4475)$ & & \\
$(-1.0000,-2.0000,1.0000,1.0000,1.0000)$ & $(-1.3073,-2.8603,-1.0470$, & $(11.3550,-9.2936)$ & $1.0 e^{-013}$ \\
\hline
\end{tabular}

\begin{tabular}{lccc}
\hline \multicolumn{5}{c}{ Now, we compare the same example with the one in [9] under the same initial point. } \\
\hline$x^{(0)}$ & $x^{*}$ & $f\left(x^{*}\right)$ & $\left\|H_{\omega^{*}}\right\|$ \\
\hline$(1.0000,2.0000,0.0000,1.0000,1.0000)$ & $(0.3077,0.5374,-0.2703$, & $(0.1336,0.2804)$ \\
& $(-2.2124,-2.0383,0.8241$, & \\
$(-1.0000,-2.0000,1.0000,1.0000,1.0000)$ & $-0.5208,8.8528)$ & $(88.3715,-19.2246)$ & $4.1 e^{-014}$ \\
\hline
\end{tabular}

Table 2: Results for Example 4.3.

\begin{tabular}{lccc}
\hline$x^{(0)}$ & $x^{*}$ & $f\left(x^{*}\right)$ & $\left\|H_{\omega^{*}}\right\|$ \\
\hline$(12.0000,3.0000)$ & $(3.0004,0.0000)$ & $(11.3566,-9.2942)$ & $1.0 e^{-012}$ \\
$(12.0000,-3.0000)$ & $(3.0003,0.0000)$ & $(11.3550,-9.2936)$ & $1.0 e^{-013}$ \\
\hline
\end{tabular}

Example 4.3. Consider

$$
\begin{array}{ll}
\min & f=\min \left\{x_{1}^{2}+x_{2}^{2}, x_{1}\right\} \\
\text { s.t. } & g_{1}(x)=-x_{2}-6 \leq 0 \\
& g_{2}(x)=x_{2}-6 \leq 0 \\
& h_{1}(x)=x_{1}-x_{2}^{2}-3=0
\end{array}
$$

(1) Since $\nabla h(x)=\left(1,-2 x_{2}\right)^{T}$, it is easy to see that the assumption $\left(\mathrm{A}_{3}\right)$ in [14] and the assumption $\left(\mathrm{C}_{3}\right)$ in [15] are not satisfied at most points in feasible set. Hence, we introduce the functions $\eta_{i}(x)(i=1,2)$ and $\beta(x)$.

(2) Let $\eta_{i}(x)=\nabla g_{i}(x)(i=1,2)$ and $\beta(x)=(10,0)^{T}$. It is easily verified that the feasible set satisfies the assumptions $\left(C_{1}\right)-\left(C_{4}\right)$. The results are shown in Table 2 .

\section{Acknowledgments}

This work was supported by National Natural Science Foundation of China (Grant no. 11171003), Key Project of Chinese Ministry of Education (Grant no. 211039), and the Jilin Province Natural Science Foundation (Grant no. 20101597).

\section{References}

[1] V. Pareto, Course Economic Politique, Rouge, Lausanne, Switzerland, 1896.

[2] J. Von Neumann and O. Morgenstern, Theory of Games and Economic Behavior, Princeton University Press, Princeton, NJ, USA, 1944.

[3] T. C. Koopmans, Activity Analysis of Production and Allocation, Wiley, New York, NY, USA, 1951. 
[4] R. B. Kellogg, T. Y. Li, and J. Yorke, "A constructive proof the Brouwer fixed-point theorem and computational results," SIAM Journal on Numerical Analysis, vol. 18, no. 4, pp. 473-483, 1976.

[5] S. Smale, "A convergent process of price adjustment and global newton methods," Journal of Mathematical Economics, vol. 3, no. 2, pp. 107-120, 1976.

[6] S. N. Chow, P. J. Mallet, and J. A. York, "Finding zero of maps: homotopy methods that are constructive with probability one," Mathematics of Computation, vol. 32, pp. 887-899, 1978.

[7] M. S. Gowda, "On the extended linear complementarity problem," Mathematical Programming Series B, vol. 72, no. 1, pp. 33-50, 1996.

[8] F. Facchinei and J. S. Pang, Finite-Dimensional Variational Inequalities and Complementarity Problems, Springer, New York, NY, USA, 2003.

[9] Y. F. Shang and B. Yu, "A constraint shifting homotopy method for convex multi-objective programming," Journal of Computational and Applied Mathematics, vol. 236, pp. 640-646, 2011.

[10] G. P. McCormick, "Projective SUMT method for convex programming," Mathematics of Operations Research, vol. 14, no. 2, pp. 203-223, 1989.

[11] R. D. C. Monteroro and I. Adler, "An extension of karmarkar type algorithm to a class of convex separable programming problems with global linear rate of convergence," Mathematics of Operations Research, vol. 15, pp. 408-422, 1990.

[12] Z. Lin, B. Yu, and G. Feng, "A combined homotopy interior point method for convex nonlinear programming," Applied Mathematics and Computation, vol. 84, no. 2-3, pp. 193-211, 1997.

[13] Z. H. Lin, D. L. Zhu, and Z. P. Sheng, "Finding a minimal efficient solution of a convex multiobjective program," Journal of Optimization Theory and Applications, vol. 118, no. 3, pp. 587-600, 2003.

[14] W. Song and G. M. Yao, "Homotopy method for a general multiobjective programming problem," Journal of Optimization Theory and Applications, vol. 138, no. 1, pp. 139-153, 2008.

[15] X. Zhao, S. G. Zhang, and Q. H. Liu, "Homotopy interior-point method for a general multiobjectuve programming problem," Journal of Applied Mathematics, vol. 2012, Article ID 497345, 12 pages, 2012.

[16] Z. S. Zhang, Introduction to Differential Topology, Beijing University Press, Beijing, China, 2002.

[17] G. L. Naber, Topological Methods in Euclidean Space, Cambridge University Press, London, USA, 1980. 


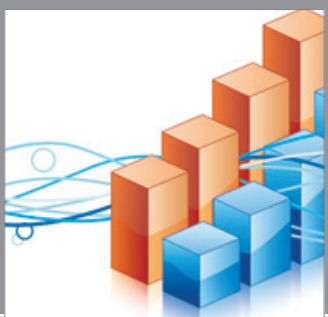

Advances in

Operations Research

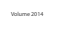

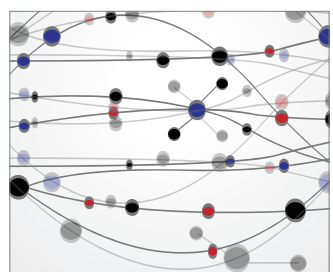

\section{The Scientific} World Journal
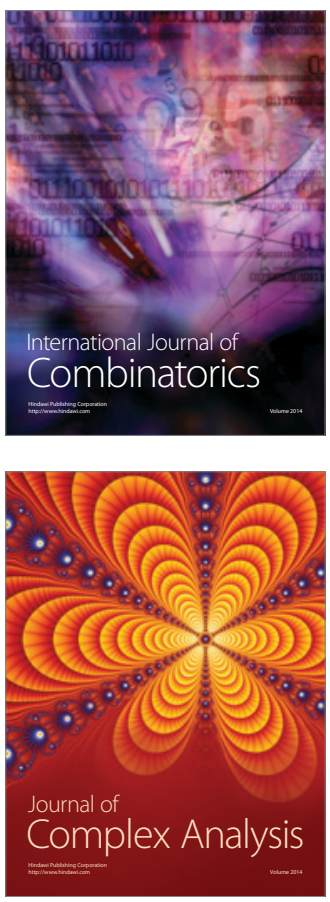

International Journal of

Mathematics and

Mathematical

Sciences
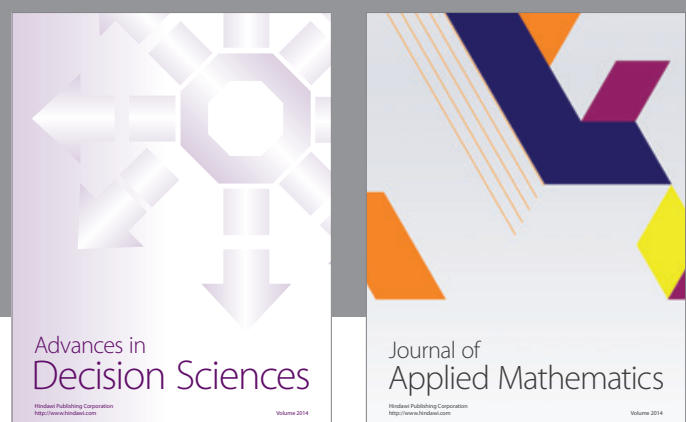

Journal of

Applied Mathematics
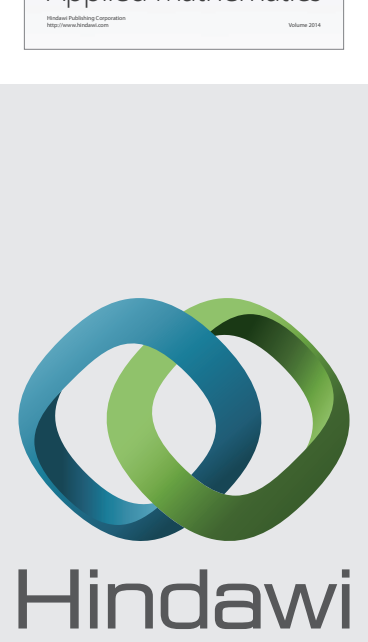

Submit your manuscripts at http://www.hindawi.com
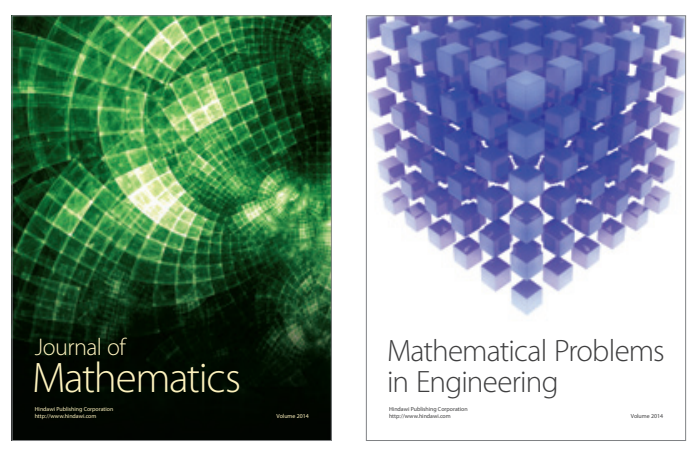

Mathematical Problems in Engineering
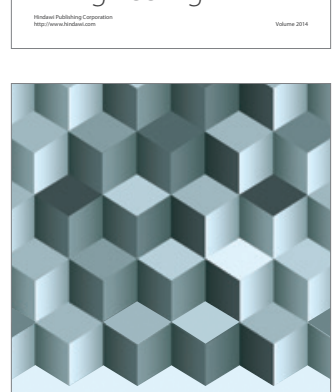

Journal of

Function Spaces
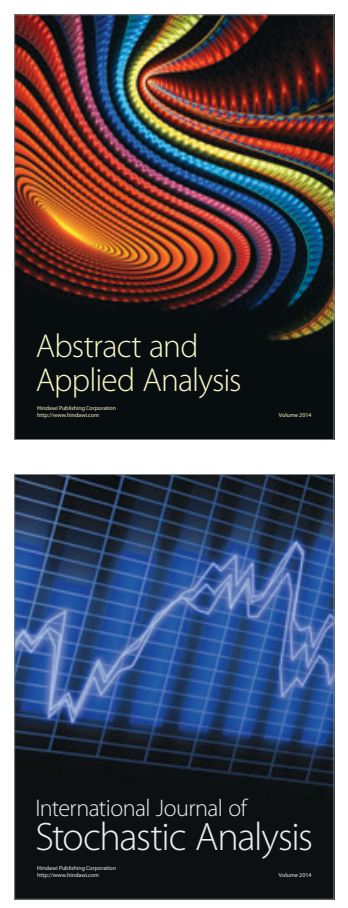

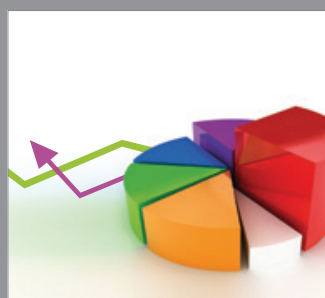

ournal of

Probability and Statistics

Promensencen
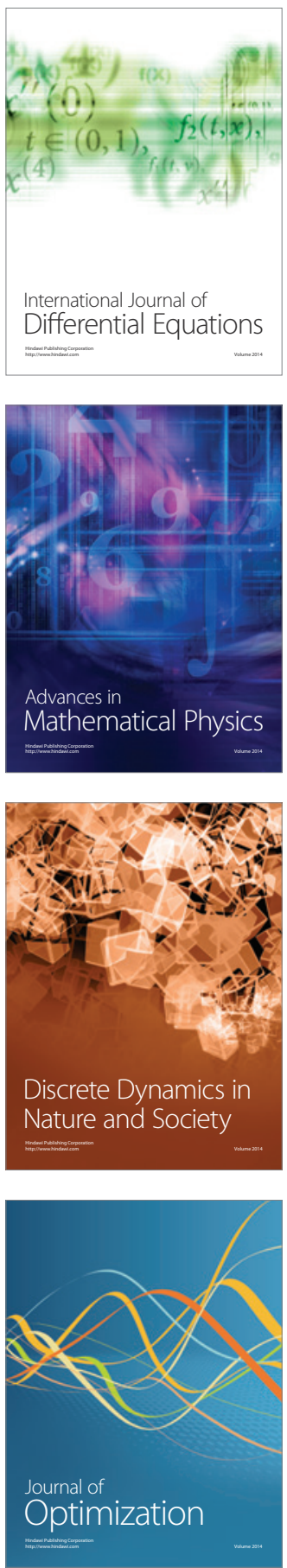